\title{
BMJ Open Investigating asthma comorbidities: a systematic scoping review protocol
}

\author{
Karim El Ferkh, ${ }^{1}$ Bright Nwaru, ${ }^{1,2}$ Chris Griffiths, ${ }^{3}$ Aziz Sheikh ${ }^{1}$
}

To cite: El Ferkh K, Nwaru B, Griffiths C, et al. Investigating asthma comorbidities: a systematic scoping review protocol. BMJ Open 2016;6: e010548. doi:10.1136/ bmjopen-2015-010548

- Prepublication history and additional material is available. To view please visit the journal (http://dx.doi.org/ 10.1136/bmjopen-2015010548).

Received 13 November 2015 Revised 30 June 2016 Accepted 11 July 2016

\section{$\underset{ }{\infty}$ CrossMark}

${ }^{1}$ Asthma UK Centre for Applied Research, Centre for Medical Informatics, Usher Institute for Population Health Sciences, The University of Edinburgh, Edinburgh, UK

${ }^{2}$ School of Health Sciences, University of Tampere, Tampere, Finland

${ }^{3}$ Asthma UK Centre for Applied Research, Centre for Primary Care and Public Health, Blizard Institute, Queen Mary University of London, London, UK

Correspondence to Karim El Ferkh; k.firikh@ed.ac.uk

\section{ABSTRACT}

Introduction: Asthma is a common long-term disorder with a number of related comorbid conditions, which may affect asthma outcomes. There is a need for greater appreciation for understanding how these comorbidities interact with asthma in order to improve asthma outcomes.

Objectives: To systematically identify and map out key asthma comorbidities.

Methods: We will systematically search the following electronic databases: MEDLINE, EMBASE, ISI Web of Science, Cumulative Index to Nursing and Allied Health Literature (CINAHL), PsycINFO and Google Scholar. Additional literature will be identified by searching the reference list of identified eligible studies and by searching the repositories of international conference proceedings, including ISI Conference Proceeding Citation Index, and ZETOC (British Library).

Dissemination: The findings from this systematic scoping review will be reported at scientific meetings and published in a peer-reviewed journal.

\section{BACKGROUND}

Asthma is typically a lifelong disease and affects over 300 million people worldwide. ${ }^{1}$ It is responsible for considerable morbidity, mortality and substantial healthcare costs. ${ }^{1} 2$ Effective self-management and pharmacotherapy leading to well-controlled asthma is the key management goal. ${ }^{3-5}$ Key indicators of successful asthma control include minimal or no symptoms, no restrictions on activities, optimal pulmonary function and minimal or no side-effects of treatment. ${ }^{3-6}$ Poor asthma control is believed mainly to result from inadequate/suboptimal treatment and problems with adherence to recommended treatments. ${ }^{45}$ An important consideration as well is the impact of comorbid diseases on asthma. ${ }^{45}$ 7-10 Many definitions exist for asthma as there is a significant proportion of heterogeneity; the definition adopted here is a doctor-diagnosed or patient-reported asthma ever.

The presence of more than one condition in a person is the widely suggested concept for comorbidity. ${ }^{11}$ The doctor-diagnosed diseases should be linked to the International

\section{Strengths and limitations of this study}

To the best of our knowledge, this is the first review undertaken to map out the full spectrum of asthma comorbidity; hence, evidence generated from the review will be important in shaping the direction of the field.

- The scoping review will be limited to the past 5 years only, but aimed to map the most contemporaneous evidence on asthma comorbidity.

- As a scoping review, formal quality assessment and risk of bias will not be undertaken on studies to be included in the review.

Classification of Diseases (ICD) regardless of their chronological occurrence (before or after the index condition: asthma). The importance of comorbidities lies not only in their presence but also in their severity that can be used to convey the concept of 'burden of disease'. ${ }^{11}$ The Charlson Index is commonly used to compare and understand the complexity of coexisting diseases. A number of conditions (eg, allergic rhinitis, gastro-oesophageal reflux disease (GORD), obesity and depression) may occur more frequently in people with asthma than in those without asthma, leading to potential additional difficulties in asthma management. ${ }^{12}{ }^{13}$ These comorbid conditions may be associated with poor asthma control, impaired health-related quality of life (HRQoL) and increased health and social care usage. ${ }^{12-19}$ The systematic identification and mapping of these comorbid conditions may lead to customised, targeted treatment, which in turn offers the potential to substantially improve outcomes in patients with asthma, and thereby reduce the need for health and social care. ${ }^{412} 1920$

There are a number of international studies investigating asthma comorbidity, impact on asthma control and HRQoL, and consequent healthcare and societal burden, but their results vary depending on the populations studied and the particular comorbid conditions that are the focus of these investigations. ${ }^{21-23}$ There is a need 
therefore for a systematic investigation into the range of asthma comorbidities. This scoping review aims to fill this need and, in addition, identify important research gaps in understanding the relationship between asthma and its comorbidities. It will not focus on studies that have looked at the association between asthma and other diseases and vice versa but on studies that have investigated the impact of other conditions on asthma outcomes.

\section{METHODS}

Eligibility criteria

Types of studies

Cohort studies, case-control studies and cross-sectional studies will be eligible for inclusion. We will exclude editorials, animal studies, reviews, randomised controlled trials (RCTs), quasi-RCTs, case studies and case series.

\section{Participants}

We are interested in studies on participants of any age with a clinical diagnosis of asthma.

\section{Outcome}

Clinical diagnosis of asthma: doctor-diagnosed asthma ever or current assessed subjectively (patient reported) or objectively (health/medical records).

\section{Asthma and the comorbidities of interest}

As this is only a scoping review, our aim is to uncover the various approaches that have been employed, including the definition of asthma that authors have employed. Therefore, restricting ourselves to a specific definition of asthma may also be restrictive to our objective of the scoping systematic review.

The comorbidities that we are interested in are those that exacerbate asthma's management and prognosis (eg, effect of asthma), regardless of whether they develop before or after asthma. We will also group comorbidities according to the Charlson Comorbidity Index, a method of categorising comorbidities of patients based on the ICD diagnosis codes. We are interested in studies on asthma comorbidities; these are likely to include, but are not limited to, allergic diseases, cardiovascular diseases, chronic obstructive pulmonary disease, autoimmune disorders (eg, type 1 diabetes), metabolic disorders (eg, type 2 diabetes, obesity), eosinophilic diseases, psychological dysfunction (anxiety, depression), hypertension, cardiovascular diseases and GORD.

\section{Search methods}

Databases

We will identify published studies from the following databases: MEDLINE, EMBASE, ISI Web of Science, Cumulative Index to Nursing and Allied Health Literature (CINAHL), PsycINFO and Google Scholar. Additional literature will be identified by searching the reference list of identified eligible studies and by searching the repositories of international conference proceedings, including ISI Conference Proceeding Citation Index, and ZETOC (British Library). Unpublished literature and ongoing studies will be identified by searching the following registries: ISI Conference Proceedings Citation Index via Web of Knowledge.

\section{Search strategy}

A highly sensitive search strategy has been developed in each of the six databases to capture the broad literature on the topic (see online supplementary appendix). Using bibliographic databases and health-oriented search engines, the search will encompass the main concept of our scoping review: asthma comorbidities.

In order to maximise the sensitivity of our search, we are not only restricting our overall strategy to topic searches in literature databases but are also taking these steps: consulting with experts in the field, looking in clinical trials registers, looking in conference proceedings, looking in grey literature such as $\mathrm{PhD}$ theses, doing forward and backward citation tracking, interrogating the websites of key organisations and hand-searching journals. We will search from March 2014 until March 2016. Although it is well known that researchers have been studying asthma as a heterogeneous disease affected by multiple comorbidities, however, the evidence on asthma and comorbidities increased exponentially in the past years. In addition, our preliminary search provided over 20000 records from one database; this 2-year time frame will enable us to achieve our objectives in a pragmatic manner.

\section{Study selection}

The articles retrieved from the search strategy will be screened according to the review inclusion and exclusion criteria. The titles and abstracts will be independently screened by two investigators for potentially eligible studies, and when there is a difference in opinions, discussions will be undertaken to reach a consensus on each article. If an agreement is not reached, a third reviewer will arbitrate. The full text versions of potentially relevant studies will be retrieved and screened independently by two reviewers; consensus will be carried out through discussions and a third reviewer will arbitrate if no agreement reached on any study. All the studies not meeting the inclusion criteria will be excluded. Study screening will be undertaken and reported according to the Preferred Reporting Items for Systematics Reviews and Meta-analyses' (PRISMA) recommendation. ${ }^{24}$

\section{Data extraction}

A customised data extraction form will be constructed to extract all relevant data from each study. The data extraction form will be piloted on a few of the eligible studies to evaluate its reliability in capturing the study data of interest. Data extraction will be undertaken 
independently by two reviewers. Any disagreements will be resolved by discussion or arbitration by a third reviewer. Descriptive summary tables will be produced to recapitulate the evidence base. The following data will be extracted:

- Author(s) and date;

- Geographical location;

- Research design;

- Aims;

- Research questions;

- Methods;

- Settings;

- Participants (N, mean age, gender if available);

- Comorbidities studied;

- Key findings;

- Research gaps identified.

An initial map will be developed to explore the main comorbidities associated with asthma. Then the findings will be synthesised in a descriptive and a narrative review and summarised in a concise table to facilitate the comparisons of different comorbidities. The replication of results and discrepancies will be investigated. The results will be then stratified according to different age groups, gender and other relevant indicators of interest. The scoping review designed will be developed according to the Levac et $a l \mathrm{~s}^{25}$ framework and the reporting will follow the PRISMA checklist. ${ }^{24}$

\section{CONCLUSIONS}

This systematic scoping review will provide a comprehensive overview of asthma comorbidities. We expect to report in the summer of 2016.

\section{ETHICS AND DISSEMINATION}

As there are no primary data collected, there will be need for formal National Health Service ethical review. The systematic scoping review will be presented at a relevant conference and be published in a peer-reviewed journal.

Contributors All authors have made substantive intellectual contributions to the development of this protocol. KEF was involved in writing this protocol. AS, CG and BN commented critically on several drafts of the manuscript. KEF, $A S$ and $B N$ were involved in conceptualising this review.

Funding This work is supported by the Chief Scientist's Office of the Scottish Government and Asthma UK as part of the Asthma UK Centre for Applied Research (AUK-AC-2012-01). BN is supported by the Farr Institute and Asthma UK Centre for Applied Research.

Competing interests None declared.

Provenance and peer review Not commissioned; externally peer reviewed.

Open Access This is an Open Access article distributed in accordance with the Creative Commons Attribution Non Commercial (CC BY-NC 4.0) license, which permits others to distribute, remix, adapt, build upon this work non-commercially, and license their derivative works on different terms, provided the original work is properly cited and the use is non-commercial. See: http://creativecommons.org/licenses/by-nc/4.0/

\section{REFERENCES}

1. Global Asthma Network. Global Asthma Report 2014, 2014.

2. WHO. Asthma Fact Sheet, 2013.

3. Global Initiative for Asthma. Global strategy for asthma management and prevention, 2012.

4. Boulet LP. Influence of comorbid conditions on asthma. Eur Respir J 2009;33:897-906.

5. Boulet LP, Boulay ME. Asthma-related comorbidities. Expert Rev Respir Med 2011;5:377-93.

6. Bateman ED, Boushey HA, Bousquet J, et al., GOAL Investigators Group. Can guideline-defined asthma control be achieved? The Gaining Optimal Asthma ControL study. Am J Respir Crit Care Med 2004;170:836-44.

7. Cazzola M, Calzetta L, Bettoncelli G, et al. Asthma and comorbid medical illness. Eur Respir J 2011;38:42-9.

8. Cazzola M, Segreti A, Calzetta L, et al. Comorbidities of asthma: current knowledge and future research needs. Curr Opin Pulm Med 2013;19:36-41.

9. De Groot EP, Duiverman EJ, Brand PL. Comorbidities of asthma during childhood: possibly important, yet poorly studied. Eur Respir J 2010;36:671-8.

10. Ledford DK, Lockey RF. Asthma and comorbidities. Curr Opin Allergy Clin Immunol 2013;13:78-86.

11. Valderas JM, Starfield B, Sibbald B, et al. Defining comorbidity: implications for understanding health and health services. Ann Fam Med 2009;7:357-63.

12. Gershon AS, Wang C, Guan J, et al. Burden of comorbidity in individuals with asthma. Thorax 2010;65:612-18.

13. Zhang T, Carleton BC, Prosser RJ, et al. The added burden of comorbidity in patients with asthma. $J$ Asthma 2009;46:1021-6.

14. Blanchette CM, Gutierrez B, Ory C, et al. Economic burden in direct costs of concomitant chronic obstructive pulmonary disease and asthma in a Medicare Advantage population. J Manag Care Pharm 2008;14:176-85.

15. Deshmukh VM, Toelle BG, Usherwood T, et al. The association of comorbid anxiety and depression with asthma-related quality of life and symptom perception in adults. Respirology 2008;13:695-702.

16. Grupp-Phelan J, Lozano P, Fishman P. Health care utilization and cost in children with asthma and selected comorbidities. J Asthma 2001;38:363-73.

17. Lehrer PM, Karavidas MK, Lu SE, et al. Psychological treatment of comorbid asthma and panic disorder: a pilot study. J Anxiety Disord 2008;22:671-83.

18. Soriano JB, Visick GT, Muellerova $\mathrm{H}$, et al. Patterns of comorbidities in newly diagnosed COPD and asthma in primary care. Chest $J$ 2005;128:2099-107.

19. Wijnhoven HA, Kriegsman DM, Hesselink AE, et al. The influence of co-morbidity on health-related quality of life in asthma and COPD patients. Respir Med 2003;97:468-75.

20. Gershon AS, Guan J, Wang C, et al. Correction: describing and quantifying asthma comorbidity: a population study. PLOS ONE 2013;8:e34967.

21. Punekar YS, Sheikh A. Establishing the sequential progression of multiple allergic diagnoses in a UK birth cohort using the General Practice Research Database. Clin Exp Allergy 2009;39:1889-95.

22. Romano A. Allergic Rhinitis and its Impact on Asthma (ARIA): achievements in 10 years and future needs. J Allergy Clin Immunol 2012.

23. Walker S, Sheikh A. Self reported rhinitis is a significant problem for patients with asthma. Prim Care Respir J 2005;14:83-7.

24. Moher D, Shamseer L, Clarke M, et al. Preferred reporting items for systematic review and meta-analysis protocols (PRISMA-P) 2015 statement. Syst Rev 2015;4:1.

25. Levac DH, Colquhoun H, O'Brien KK. Scoping studies: advancing the methodology. Implement Sci 2010;5:1-9. 